\title{
New Model for Effect of Fringing Fields on Radius of Circular Microstrip Antenna
}

\author{
S. K. Bhatnagar \\ Electronics and Communication Engineering Department, Swami Keshvanand Institute of Technology, \\ Management and Gramothan, Jaipur-302017 (INDIA) \\ Email: bhatnagar_skb@yahoo.de \\ Received 04.02.2021, received in revised form 18.03.2021, accepted 04.04.2021
}

\begin{abstract}
This paper presents a model for effect of fringing fields on the radius of Circular Micro Strip Antenna (CMSA). The model is straight forward, simple and accurate for determining the physical radius of the circular patch. It is proposed that the extension in physical radius of CMSA is directly proportional to the physical radius itself and also to the normalized thickness of the dielectric substrate. The new model gives good results without any iteration. Novel results are that for all CMSA (1) the ratio of extension in physical radius to physical radius is equal to $H$ (2) ratio of fringing field area to physical area of the patch is $2 \mathrm{H}$ (3) ratio of extension in physical radius to the height of the substrate is a constant Anm/2 $\pi$. Here $H$ is the normalized substrate thickness. Normalization is done with respect to guide wavelength. Anm is the mth zero of the derivative of the Bessel function of the order $n$. Large number of CMSA have been designed and simulated to validate the new thinking. Some typical data and simulation results have been incorporated in this paper.
\end{abstract}

Keywords- Bhatnagar's Postulate, Circular Microstrip Antenna, Design, Fringing Fields, Guide Wavelength, Physical Dimensions, Resonant frequency

\section{INTRODUCTION}

Design of microstrip antennas is always complicated due to the effects of the fringing fields. As a result, the patch appears to have electrical dimensions (called effective dimensions) that are slightly different from its physical dimensions. The user wants to know physical dimensions of the patch. But the design formulae exist for effective dimensions of the patch. The patch may have any shape and size. However, the dimensions of the patch and its shape determine the characteristics of the resulting antenna. Several iterations are therefore normally done to optimize these. Generally this process is repeated every time a new design is to be made.

Fig. 1. shows basic geometry of a circular microstrip antenna (CMSA). An antenna resonating at a frequency, $f_{r}$, is to be designed with circular patch geometry on a substrate of thickness $h$ and dielectric constant $\varepsilon_{r}$. The problem is to determine the value of the physical radius, $a_{p}$, of the antenna patch. The classical formulae for such an antenna are complex and interwoven. To account for the effects of fringing fields 'effective radius' and 'effective dielectric constant' were conceptualized. Formulae were evolved by semiempirical and curve fitting methods. Better results were obtained by considering dynamic dielectric constant, total capacitance and fringing capacitance [1]. Artificial Neural Networks (ANN) have also been used for determining $a_{p}$ [2]. Researchers in antenna area often design CMSA and need to know the physical radius of the patch. Extension in this radius, fringing field area and relation of this area with the patch area are all required many times. This paper seeks to find a simple model for estimating $a_{p}$ and extension in $a_{p}$ due to fringing fields. Further investigations have been done for finding fringing field area and its relation with patch area. These are of great importance for the Antennas and Propagation Community in designing such antennas and also in controlling their dimensions. As the antennas are shrinking to fit in a chip, the antenna designers need precise solutions.

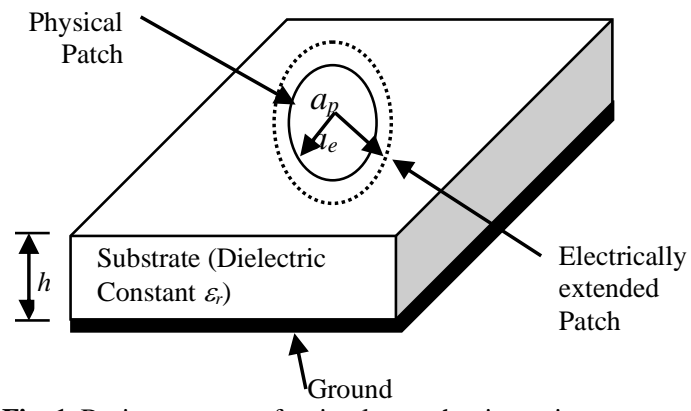

Fig. 1. Basic geometry of a circular patch microstrip antenna showing physical patch and it's electrical extension [3]

The effective radius of the patch, $a_{e}$, is given by

$$
a_{e}=\frac{A_{n m * c}}{2 \pi f_{r} \sqrt{\varepsilon_{r}}}
$$

where $A_{n m}$ is the $\mathrm{m}^{\text {th }}$ zero of the derivative of the Bessel function of the order $\mathrm{n}$ and $\mathrm{c}$ is the speed of light in free space.

The relation between $a_{e}$ and $a_{p}$ is

$$
\begin{aligned}
& a_{e} a_{p} *\left[1+\frac{2 h}{\pi a_{p} \varepsilon_{r}}\left(\ln \left\{\frac{\pi a_{p}}{2 h}\right\}+1.7726\right)\right]^{0.5} \\
& \text { for } \frac{a_{p}}{h} \gg>1
\end{aligned}
$$


For the lowest order mode $A_{n m}=1.84118$. Also $\mathrm{c}=$ $3 * 10^{10} \mathrm{~cm} / \mathrm{s}$. This gives

$a_{p}=\frac{8.791}{f_{r} \sqrt{\varepsilon_{r}}} *\left[1+\frac{2 h}{\pi a_{p} \varepsilon_{r}}\left(\ln \left\{\frac{\pi a_{p}}{2 h}\right\}+1.7726\right)\right]^{-0.5}$ (4)

Here $a_{p}$ and $h$ are in $\mathrm{cm}$ and $f_{r}$ is in GHz.

\section{THE NEW APPROACH}

\subsection{Methodology}

Statistical analysis approach has been used. Large amount of data was generated and analyzed to conclude a model that answers most of the questions. Classical formulae [3] have been used for data generation. Each of the basic variables $\varepsilon_{r}, h$ and $f_{r}$ has been varied over the normal range to estimate the values of $a_{p}, a_{e}$ and $H$. The parameter $H$ is given by

$$
H=\frac{h}{\lambda_{g}}=\frac{1}{c} h f_{r} \sqrt{\varepsilon_{r}}
$$

$\lambda_{g}$ is the guide wavelength. The data was analyzed to investigate extension in $a_{p}$ i.e. $\left(a_{e}-a_{p}\right)$ and related issues. No trend was visible. To derive meaningful conclusions the data was partitioned into groups on the basis of constant $H$ value. It has been shown earlier that for rectangular patch MSA, the extension in physical length of the patch is directly proportional to $H$ and also to the effective length of the patch (Bhatnagar's Postulate) [4]. This theory has been extended to equilateral triangular MSA. It has been argued that the effective length and effective dielectric constant are conceptual parameters only. The real parameters that can be measured are the physical length and the dielectric constant. Therefore, the model should be based on these [5]. The methodology was to investigate whether similar thing exists for circular MSA also

\subsection{Results}

Extension in $a_{p}$ was investigated as a function of $a_{p}$. The classical model Data was plotted as in Fig. 2. In this figure, for Series $1, H=0.010$, the plot is a straight line given by the equation $\left(a_{e}-a_{p}\right)=0.0102$ $a_{p}$; for Series $2, H=0.015$, the plot is a straight line given by the equation $\left(a_{e}-a_{p}\right)=0.0142 a_{p}$ and for Series $3, H=0.020$, the plot is a straight line given by the equation $\left(a_{e}-a_{p}\right)=0.0179 a_{p}$;

All the lines are passing through the origin. These can be represented by the general equation

$$
a_{e}-a_{p}=H a_{p}
$$

This is a very important conclusion. It indicates that the Bhatnagar's postulate is valid for CMSA also. It can then be stated that "For a circular microstrip antenna, the extension in physical radius, due to fringing fields, is proportional to the physical radius and also to the normalized substrate thickness".
Fig. 2. Dependence of extension in physical radius on physical radius for thin substrates $(\mathrm{H} \leq 0.02)$

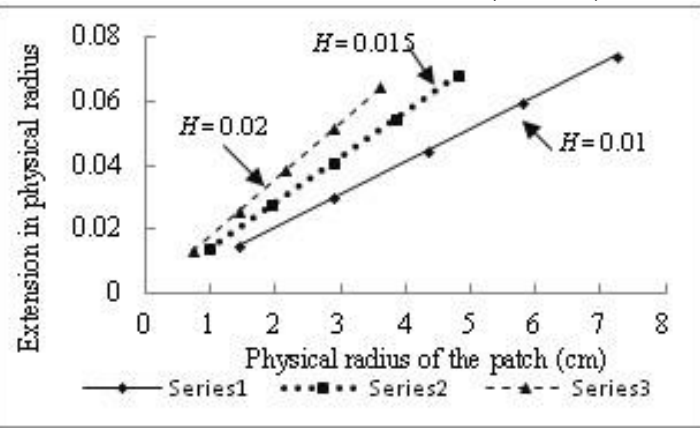

As $H \ll 1$, using Binomial Theorem (6) can be rewritten as

$$
a_{p}=\frac{a_{e}}{1+H}=\left(1-H+H^{2}-H^{3}+\ldots\right) a_{e}
$$

Further using (1)

$$
a_{p}=\frac{A_{n m} * c}{2 \pi f_{r} \sqrt{\varepsilon_{r}}}-\frac{A_{n m} * h}{2 \pi}
$$

For the lowest order mode $\mathrm{TM}_{11}$ (7) reduces to

$$
a_{p}=\frac{8.791}{f_{r} \sqrt{\varepsilon_{r}}}-0.293 * h
$$

This very simple model determines the physical radius directly from the basic parameters $f_{r}, \varepsilon_{r}$ and $h$. This is a one step calculation. All earlier methods have at least two steps. There is no need for calculating any effective dielectric constant. The agreement between the new and the classical results is very good.

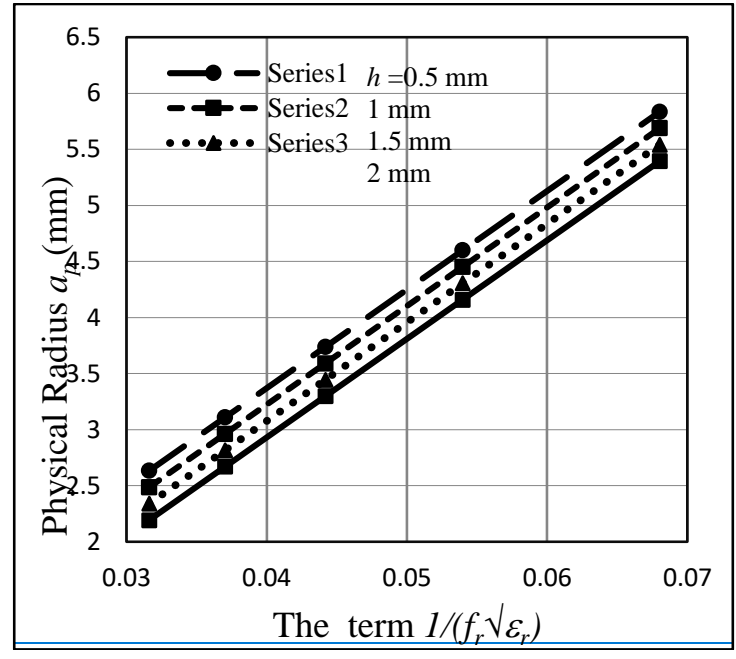

Fig. 3. Graphical tool for directly estimating the physical radius of circular patch.

\section{VALIDATION}

Calculations were done using new formulae as well as classical formulae. Fig. 3. shows that the graph between $a_{p}$ and $1 / f_{r} \sqrt{ } \varepsilon_{r}$ is a straight line for constant value of $h$. A change in $h$ shifts the line parallel to itself. This validates the new thinking. Using this plot $a_{p}$ can be directly obtained. Thus Fig. 3. can be used as a graphical tool for determining $a_{p}$. 
No need for any calculation. Just find inverse of $f_{r} \vee_{\varepsilon_{r}}$. Select the curve corresponding to the desired substrate thickness $h$ (for the value corresponding to given $f_{r}$ and $\varepsilon_{r}$ ) and read $a_{p}$ directly from the tool. Simplest, quickest and accurate method for determining $a_{p}$.

While keeping $f_{r}$ and $\varepsilon_{r}$ fixed, relation between Extension in Physical radius, $\left(a_{e}-a_{p}\right)$, and the substrate thickness, $h$, was investigated. From (7) it can be deduced that

$\left(a_{e}-a_{p}\right)=\left(A_{n m} / 2 \pi\right) h$

Thus the new formula shows a linear relationship between the extension in physical radius and the substrate thickness. Classical formulae also indicate a linear relationship between these two quantities. Agreement between the old and the new formulae is very good.

For different combinations of the basic parameters $f_{r}, h$ and $\varepsilon_{r}$, the ratio $a_{e} / a_{p}$ and $H$ were computed by classical formulae and the new formula. The resulting plot is depicted in Fig. 4. It is found that for all values of $\mathrm{H}$, the ratio $a_{e} / a_{p}$ is always equal to $(1+H)$. This is a very important part of the new postulate that the extension in the physical radius is directly proportional to $H$.

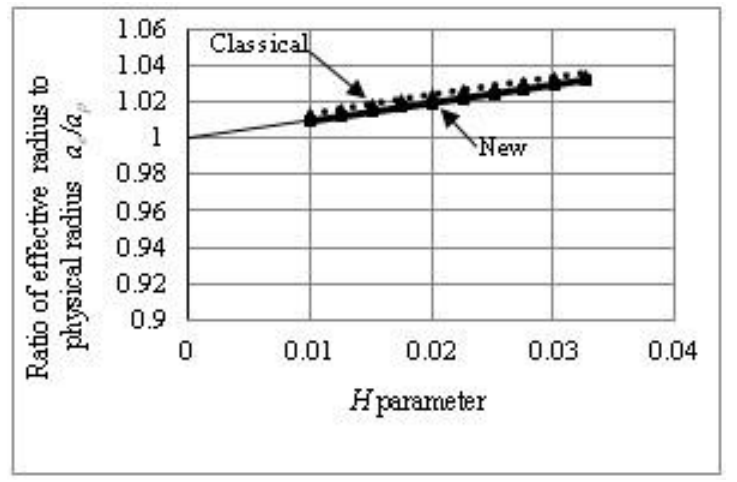

Fig. 4. Dependence of radius ratio on $\mathrm{H}$ for CMSA. Ratio of effective radius to physical radius is equal to $(1+\mathrm{H})$

\section{SIMULATIONS}

Very large number of CSMA have been designed for various values of $h$ and $\varepsilon_{r}$ for resonant frequency varying from $1 \mathrm{GHz}$ to $10 \mathrm{GHz}$. This leads to variations in $a_{e}$ and hence $a_{p}$. The antenna structure was designed. Physical radius $a_{p}$ was calculated by the new formula (8) as well as by the classical formula. The structure was simulated, using HFSS software, for classical as well as new designs. A typical plot of $\mathrm{S}_{11}$ against $f_{r}$ for designed frequency of $2.0 \mathrm{GHz}$ is shown in Fig. 5. The classical formula gave $a_{p}=28.48 \mathrm{~mm}$ resulting in $f_{r}$ $=2.0996 \mathrm{GHz}$ while the new formula gave $a_{p}=29.4$ $\mathrm{mm}$ and $f_{r}=2.0416 \mathrm{GHz}$.

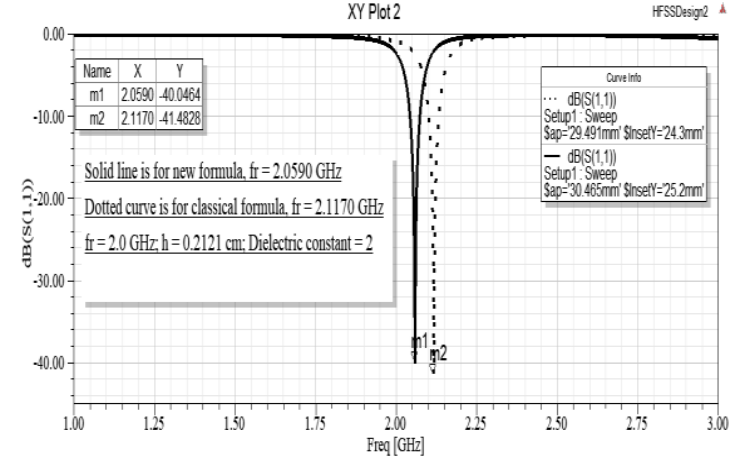

Fig. 5. Comparison of simulations based on new as well as classical formulae

\section{DISCUSSIONS}

Classical method of determining $a_{p}$ is a 2 step procedure. In the first step $a_{e}$ is determined using (1). In the second step this value of $a_{e}$ is used in (4) to find $a_{p}$. This second step is not straight forward. Equation (4) is of the form $x=f(x)$. Initially $a_{p}=a_{e}$ is assumed as an approximate value. Then the iterations are done till a stable value of $a_{p}$ is reached [3].

Table 1 Reverse modeling for prediction of physical radius

\begin{tabular}{|c|c|c|c|c|c|c|}
\hline \multirow{2}{*}{$\begin{array}{c}\boldsymbol{f}_{\boldsymbol{r}} \\
(\mathbf{G H z})\end{array}$} & \multirow{\varepsilon}{*}{$\boldsymbol{h}(\mathbf{c m})$} & $\begin{array}{c}\text { Physical Radius } \boldsymbol{a}_{\boldsymbol{p}} \\
(\mathbf{c m})\end{array}$ & \multicolumn{2}{c|}{$\begin{array}{c}\text { Simulated } \boldsymbol{f}_{\boldsymbol{r}} \\
(\mathbf{G H z})\end{array}$} \\
\cline { 5 - 8 } & & & Classical & New & Classical & New \\
\hline 2 & 2 & 0.2121 & 2.9491 & 3.0465 & 2.1170 & 2.0590 \\
\hline 9 & 3 & 0.0962 & 0.5254 & 0.5358 & 9.540 & 9.360 \\
\hline 10 & 4 & 0.075 & 0.4167 & 0.4176 & 10.491 & 10.4700 \\
\hline 7 & 5 & 0.0958 & 0.5381 & 0.5336 & 7.3080 & 7.3750 \\
\hline 6 & 6 & 0.1021 & 0.5772 & 0.5684 & 6.254 & 6.315 \\
\hline 5 & 7 & 0.1134 & 0.6444 & 0.6313 & 5.178 & 5.221 \\
\hline 4 & 8 & 0.1326 & 0.7564 & 0.7382 & 4.1360 & 4.191 \\
\hline 3 & 9 & 0.1667 & 0.9537 & 0.928 & 3.0840 & 3.14 \\
\hline 8 & 10 & 0.0593 & 0.3401 & 0.3301 & 8.2670 & 8.3890 \\
\hline 1 & 11 & 0.1809 & 2.6248 & 2.5973 & 1.0160 & 1.0270 \\
\hline
\end{tabular}

Physical radius was calculated by this classical method as well as by the new method proposed in this paper. This was done for resonant frequencies from $1 \mathrm{GHz}$ to $10 \mathrm{GHz}$, dielectric constant from 2 to 11. The substrate thickness varied accordingly from $0.05 \mathrm{~cm}$ to $0.25 \mathrm{~cm}$. Few results are summarized in Table I. It can be seen that the $a_{p}$ values obtained by the two methods match very well. Simulations were done for each and every value of $a_{p}$. Simulated values of $f_{r}$ indicate that nearly the same result is obtained by these two vastly different methods. Results were compared with earlier published work also.

Table II gives comparison with values obtained by Artificial Neural Networks (ANN) and measured values as per [1]. The matching between the ANN and the new formula is excellent. 


\section{A. Fringing Field Area and $H$}

For calculating effective permittivity, the existing work [3], [7] assumes that the extension in $a_{p}$ is equal to the substrate thickness, $h$, and then computes the ratio of fringing field area to the area of the patch. The new model predicts this extension to be $0.293 h$. The novelty of the proposed model is that there is no need for such assumptions and calculations. The desired ratio is independent of the patch area and fringing field area. This ratio is equal to $2 H$. This is shown below:

As per the new model $a_{e} / a_{p}=1+H$, therefore,

$$
\begin{gathered}
\frac{\text { Fringing area }}{\text { Patch area }}=\frac{\text { Extended area }- \text { Patch area }}{\text { Patch area }} \\
=\frac{\text { Extended area }}{\text { Patch area }}-1 \\
\frac{\text { Fringing area }}{\text { Patch area }}=\frac{\pi a_{e}^{2}}{\pi a_{p}^{2}}-1=(1+H)^{2}-1=2 H
\end{gathered}
$$

since $H \ll 1, H^{2}$ can be neglected.

Table 2 Forward modeling for the prediction of resonant frequency

\begin{tabular}{|c|c|c|c|c|c|}
\hline \multirow{2}{*}{$\begin{array}{c}\boldsymbol{a}_{\boldsymbol{p}} \\
(\mathbf{c m})\end{array}$} & \multirow{2}{*}{$\begin{array}{c}\boldsymbol{c} \mathbf{c m}) \\
\end{array}$} & \multirow{\varepsilon}{*}{$\boldsymbol{\varepsilon}$} & \multicolumn{3}{|c|}{$\boldsymbol{f}_{\boldsymbol{r}}(\mathbf{G H z})$} \\
\hline 3.493 & 0.1588 & 2.5 & 1.5708 & 1.57 & 1.555 \\
\hline 1.27 & 0.0794 & 2.59 & 4.2238 & 4.07 & 4.175 \\
\hline 3.493 & 0.3175 & 2.5 & 1.5504 & 1.51 & 1.522 \\
\hline 13.894 & 1.27 & 2.7 & 0.375 & 0.378 & 0.37 \\
\hline 4.95 & 0.235 & 4.55 & 0.8212 & 0.825 & 0.827 \\
\hline 3.975 & 0.235 & 4.55 & 1.0191 & 1.03 & 1.027 \\
\hline 2.99 & 0.235 & 4.55 & 1.3473 & 1.36 & 1.358 \\
\hline 2 & 0.235 & 4.55 & 1.9921 & 2.003 & 2.009 \\
\hline 1.04 & 0.235 & 4.55 & 3.7167 & 3.75 & 3.744 \\
\hline 0.77 & 0.235 & 4.55 & 4.913 & 4.945 & 4.938 \\
\hline
\end{tabular}

\section{B. Significance of $\boldsymbol{H}$}

As in the case of rectangular patch, for the circular patch also the new thinking is that the basic parameter controlling the antenna characteristics is
$H$. It embodies the effects of $f_{r}, \varepsilon_{r}$ and $h$ into a single parameter. All the formulae should be based on $H$. This should then lead to simplification and uniqueness of results. Any variation in $h, \varepsilon_{r}$ or $f_{r}$ will change $H$ and will thus be reflected in the concerned formulae. The important thing that comes up is that any change in either $h$ or $\varepsilon_{r}$ or $f_{r}$ can be offset by suitably changing the other parameters so that $H$ remains constant. All other quantities may then remain unchanged. This is the uniqueness of $H$.

\section{REFERENCES}

[1] N. Kumprasert and W. Kiranon, "Simple and accurate formula for the resonant frequency of the circular microstrip disk antenna", IEEE Transactions on Antennas and Propagation, Vol. 43, No. 11 (November 1995) pp 1331

[2] S. P. Gangwar, R. P. S. Gangwar, B. K. Kanaujia and Paras, "Resonant frequency of circular microstrip antenna using artificial neural networks" Indian Journal of Radio and Space Physics, Vol. 37, (June 2008), pp 204-208

[3] R. Garg, P. Bhartia, I. Bahl and A. Ittipiboon, "Circular disk and ring antennas" in "Microstrip Antenna Design Handbook" Artech House, Boston, (2001)

[4] D. Mathur, S. K. Bhatnagar and V. Sahula, "Quick estimation of rectangular patch antenna dimensions based on equivalent design concept" IEEE Antennas and Wireless Propagation Letters, Vol 13,(2014), pp 1469 - 1472. Available online since 01 July 2014

[5] S. K. Bhatnagar " New design formulae for equilateral triangular microstrip antenna" International Research Journal of Engineering and Technology, Volume: 07 Issue: 11(Nov 2020) pp 946-952

[6] A. K. Verma and Nasimuddin, "Analysis of circular microstrip antenna on thick substrate" J. Microwave and Optoelectronics Vol 2, No. 5, (July 2002), pp 30 -38

[7] P. Mythili and A. Das "Simple approach to determine resonant frequencies of microstrip antennas" IEE Proc. Microw. Antennas Propag., Vol. 145, NO 2 (April 1998) pp 159-162 